\title{
Recovering Shape Characteristics on Near-flat Specular Surfaces
}

\author{
Yuanyuan Ding and Jingyi Yu \\ Department of Computer and Information Sciences \\ University of Delaware \\ Newark, DE 19716, USA \\ \{ding, yu\}@cis.udel. edu
}

\begin{abstract}
We consider the problem of capturing shape characteristics on specular (refractive and reflective) surfaces that are nearly flat. These surfaces are difficult to model using traditional methods based on reconstructing the surface positions and normals. These lower-order shape attributes provide little information to identify important surface characteristics related to distortions. In this paper, we present a framework for recovering the higher-order geometry attributes of specular surfaces. Our method models local reflections and refractions in terms of a special class of multiperspective cameras called the general linear cameras (GLCs). We then develop a new theory that correlates the higher-order differential geometry attributes with the local GLCs. Specifically, we show that Gaussian and Mean Curvature can be directly derived from the camera intrinsics of the local GLCs. We validate this theory on both synthetic and real-world specular surfaces. Our method places a known pattern in front of a reflective surface or beneath a refractive surface and captures a distorted image on the surface. We then compute the optimal GLC using a sparse set of correspondences and recover the curvatures from the GLC. Experiments demonstrate that our methods are robust and highly accurate.
\end{abstract}

\section{Introduction}

Modeling and reconstructing 3D specular (reflective and refractive) surfaces is a challenging problem and has drawn considerable attention in recent years. It can be used in numerous applications, from the Fluid Mechanics perspective as well as from the Computer Graphics and Vision perspective. For example, the recovered fluid surfaces can provide direct quantitative comparisons and validation of existing fluid models [5, 24]. In catadioptric mirror design, the recovered mirror geometry can help reduce distortions. Captured fluid surfaces have also been directly integrated into

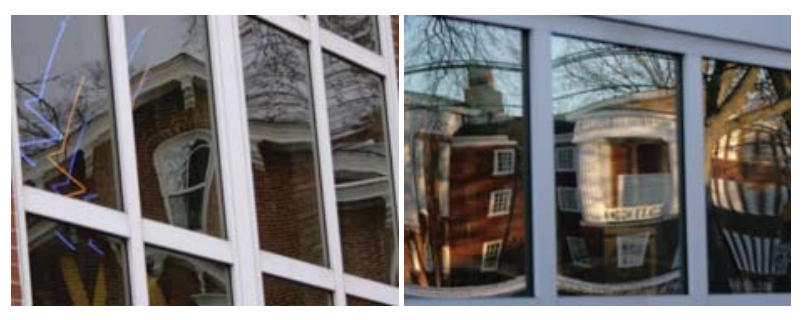

Figure 1. Distortions observed on near-flat window surfaces.

animations and film footage to reproduce realistic phenomena [4].

In this paper, we focus on a special class of specular surfaces: near-flat specular surface. These surfaces are particularly difficult to model using conventional methods that are based on recovering the lower-order geometry attributes such as the surface positions or normals. For near-flat surfaces, these attributes provide little information to identify important surface characteristics related to distortions. A typical example is the image observed on a window as shown in Figure 1. Although the surface is highly planar and the normals are approximately the same, the reflection image still exhibits complex distortions. Konderink have shown that these distortions are caused by the higher-order differential geometry attributes such as the curvature [10].

We present a new framework for directly recovering the higher-order shape characteristics of near-flat specular surfaces. We use a special class of multiperspective cameras called the general linear cameras (GLCs) to model the local reflections and refractions. The GLC model characterizes all 2D planes in the ray space as one of the eight multiperspective cameras. This analysis allows us to identify the ray structure at each point on the surface in terms of the GLC. We then derive a new ray-curvature theory to correlate the higher-order differential geometry attributes with the GLC model. Specifically, we show the Gaussian and the Mean Curvature of the specular surface can be directly computed using the camera intrinsics of the local GLC.

We validate our new theory on both synthetic and realworld surfaces. We place a known pattern in front of a re- 
flective medium or beneath a refractive medium and captures a distorted image on the surface of the medium. We then compute the optimal GLC using a sparse set of correspondences and recover the curvatures from the GLC. Experiments demonstrate that our methods are robust and highly accurate.

\section{Related Work}

Reconstructing highly specular surfaces is one of the few remaining open problems in computer vision[8]. Many single-image-based reconstruction methods have followed a shape-from-distortion approach by analyzing the distortion of a known pattern placed near the surface. Sanderson et al. [15] have shown that it is impossible to completely reconstruct the surface from a single image. Therefore, many constraints in different forms have been introduced to eliminate this ambiguity, including the planarity assumption [7, 13], surface smoothness prior [16], surface integrability constraints[19], and the use of special optics $[23,20]$. A common problem in these methods is that they are highly sensitive to radiometric inconsistency, calibration errors, and surface perturbations.

Alternatively, stereo or multi-view reconstruction methods have been used to more robustly and accurately recover the specular surfaces. Sanderson et al. [15] proposed to use a stereo camera configuration for resolving the specular ambiguity. Blake [1] measured the variations of specularities from different viewing directions to determine the differential properties of the surface. Bonfort and Sturm [2] used multiple-view geometry to build a volumetric reconstruction of mirror surfaces. Recently, Morris and Kutulakos [12] used a stereo-camera to recover the refractive disparity on complex and dynamic fluid surfaces. Bonfort and Sturm [3] used two images of the same pattern in two different positions to reconstruct specular surfaces. Kutulakos and Steger further used light-path triangulation to handle multiple reflections and refractions [11].

In this paper, we focus on modeling a special class of specular surfaces: near-flat surfaces such as windows and relatively flat fluid surfaces. Reconstructing these surfaces is particularly challenging for two reasons. First, most existing methods are designed to recover the lower-order shape attributes of the surface. However, the most important shape characteristics of near-flat surfaces are the high-order surface attributes such as the curvatures. These attributes determine the unique appearance of distortions [22], the apperance of silhouettes [9], and the motion of specular highlights [10]. Second, the surfaces recovered by traditional methods are usually noisy due to calibration errors and surface perturbations. Hence, it is impractical to use these lower-order attributes to directly estimate the higher-order attributes.

We present a new ray-curvature theory to model the higher-order geometry attributes on specular surfaces by analyzing the local ray structure. Swaminanthan and Nayar posposed to use the caustic surfaces to model the ray geometry on catadioptric mirrors $[18,17]$. Caustic surfaces are formed by the focii of the rays. When the camera moves off the focus of the mirror, the caustic surface quickly evolves into complicated shapes with discontinuities and becomes difficult to model [18, 2]. Furthermore, it is unclear how the caustic surfaces of rays are related to surface differential geometry. Recently, Yu and McMillan proposed a ray geometry analysis similar to ours to model reflection distortions. They focused on characterizing the local distortions as one of the multiperspective cameras whereas we aim to recover the surface characteristics using the local ray geometry.

Before proceeding, we explain our notation. Superscripts, such as $d^{x}, d^{y}$, and $d^{z}$ represent the $x$ and $y$ and $z$ component of a point or vector. Subscripts, such as $z_{x}$ and $z_{y}$ represent the first-order partial derivatives of $z$ with respect to $x$ and $y$. And similarly, $z_{x x}$ refers to the secondorder partial derivative of $z$ with respect to $x$.

\subsection{General Linear Cameras}

Our ray-curvature theory is based on modeling local reflections and refractions as multiperspective cameras. Yu and McMillan [21] recently proposed the general linear camera (GLC) model to unify traditional perspective, orthographic, and multiperspective cameras models. In the GLC framework, every ray is parameterized by its intersections with the two parallel planes, where $[s, t]$ is the intersection with the first and $[u, v]$ the second. This parametrization is often called a two-plane parametrization (2PP). Except for those rays parallel to the two planes, $2 \mathrm{PP}$ uniquely represents each ray by mapping it to a point in a four-dimensional ray space.

In this paper, we simplify the GLC model by substituting $\sigma=s-u$ and $\tau=t-v$ and we will use this $[\sigma, \tau, u, v]$ parametrization to represent rays. We also assume the default $u v$ plane is at $z=0$ and the st plane at $z=1$, thus $[\sigma, \tau, 1]$ represents the direction of the ray.

A GLC is defined as the affine combination of three rays, or, as a plane in the $4 \mathrm{D}$ ray space,

$$
\begin{aligned}
G L C & =\left\{r: r=\alpha \cdot\left[\sigma_{1}, \tau_{1}, u_{1}, v_{1}\right]+\beta \cdot\left[\sigma_{2}, \tau_{2}, u_{2}, v_{2}\right]\right. \\
& \left.+(1-\alpha-\beta) \cdot\left[\sigma_{3}, \tau_{3}, u_{3}, v_{3}\right], \forall \alpha, \beta\right\}
\end{aligned}
$$

Most well-known multiperspective cameras, such as pushbroom, cross-slit, and the linear oblique cameras are GLCs. Furthermore, [21] provides a characteristic equation to determine the type of the multiperspective camera for any GLC specification given three rays:

$$
\left|\begin{array}{lll}
u_{1}+\lambda \cdot \sigma_{1} & v_{1}+\lambda \cdot \tau_{1} & 1 \\
u_{2}+\lambda \cdot \sigma_{2} & v_{2}+\lambda \cdot \tau_{2} & 1 \\
u_{3}+\lambda \cdot \sigma_{3} & v_{3}+\lambda \cdot \tau_{3} & 1
\end{array}\right|=0
$$




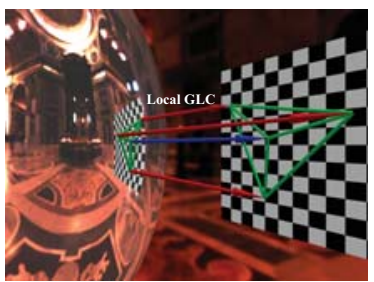

(a)

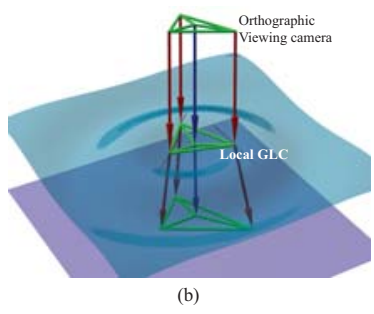

(b)
Figure 2. We map the reflection (a) and refractions rays (b) into the ray space and use the GLC to analyze the local ray structure.

Equation (1) yields to a quadratic equation in $\lambda$, and the number of solutions determines how many singularities (lines or points) all rays in the GLC simultaneously pass through. A total of eight GLC types describe all 2D linear manifolds in the ray space [21].

To use the GLCs to locally model reflections and reflections, we assume all rays reflected and refracted off the surface can be parameterized as:

$$
\Sigma(x, y)=[\sigma(x, y), \tau(x, y), u(x, y), v(x, y)]
$$

At every point in this ray manifold, we can compute a ray tangent plane with two spanning vectors $\overrightarrow{d_{1}}$ and $\overrightarrow{d_{2}}$ :

$$
\overrightarrow{d_{1}}=\left[\sigma_{x}, \tau_{x}, u_{x}, v_{x}\right], \quad \overrightarrow{d_{2}}=\left[\sigma_{y}, \tau_{y}, u_{y}, v_{y}\right]
$$

We can then compute the ray characteristic equation of the local GLC at $\Sigma(x, y)$ by choosing three rays $\Sigma(x, y)$, $\Sigma(x, y)+\overrightarrow{d_{1}}$, and $\Sigma(x, y)+\overrightarrow{d_{2}}$ as:

$$
\left|\begin{array}{ccc}
u+\lambda \sigma & v+\lambda \tau & 1 \\
\left(u+u_{x}\right)+\lambda\left(\sigma+\sigma_{x}\right) & \left(v+v_{x}\right)+\lambda\left(\tau+\tau_{x}\right) & 1 \\
\left(u+u_{y}\right)+\lambda\left(\sigma+\sigma_{y}\right) & \left(v+v_{y}\right)+\lambda\left(\tau+\tau_{y}\right) & 1
\end{array}\right|=0
$$

yielding to the quadratic equation

$$
A \lambda^{2}+B \lambda+C=0
$$

where

$$
\begin{aligned}
& A=\sigma_{x} \tau_{y}-\sigma_{y} \tau_{x} \\
& B=\sigma_{x} v_{y}-\sigma_{y} v_{x}-\tau_{x} u_{y}+\tau_{y} u_{x} \\
& C=u_{x} v_{y}-u_{y} v_{x}
\end{aligned}
$$

We call Equation (4) the local GLC characteristic equation and its coefficients $A, B$, and $C$ are the camera intrinsics to the local GLC. Figure 2 illustrates the general process for analyzing the reflection and refraction rays.

\section{Ray-Curvature Theory}

We start by mapping the reflected or refracted rays into the $[\sigma, \tau, u, v]$ ray space. At each point on the surface, we compute the reflected or refracted ray using Snell's Law and then project it onto the $u v$ and $s t$ ray parametrization plane, as shown in Figure 3. Assume the surface is parameterized in $x$ and $y$, all the reflected or refracted rays can be mapped onto a ray manifold as shown in Equation (2). To analyze the local ray geometry, we can then use the GLC to model the local behavior of rays using the ray characteristic equation (4).

At each point $\dot{O}$ on the specular surface, we choose the $u v$ parametrization plane to be the tangent plane of the surface at $\dot{O}$. Furthermore, we assume $\dot{O}$ is the origin of the $u v$ plane and $z$-axis coincides with the normal direction at $\dot{O}$ as shown in Figure 3(a).

We assume the surface is locally a Monge function $z(x, y)$ with respect to the $u v$ plane. Therefore, the surface normal of points in the local neighborhood of $\dot{O}$ can be computed as:

$$
\vec{n}=\left[-z_{x},-z_{y}, 1\right]
$$

In this paper, we also assume that for both reflective and refractive surfaces, the viewing camera is an orthographic camera that has direction:

$$
\vec{i}=\left[d^{x}, d^{y},-1\right]
$$

\subsection{Reflection Curvature Analysis}

To compute the local reflection ray manifold, we can compute the reflected direction $r$ as:

$$
\vec{r}=\vec{i}-2(\hat{n} \cdot \vec{i}) \hat{n}
$$

where $\hat{n}$ is the normalized $\vec{n}$ as

$$
\hat{n}=\left[-\frac{z_{x}}{\sqrt{\beta}},-\frac{z_{y}}{\sqrt{\beta}}, \frac{1}{\sqrt{\beta}}\right], \quad \beta=z_{x}^{2}+z_{y}^{2}+1
$$

Substituting Equation (8) and (10) into Equation (9), we have:

$$
\begin{aligned}
& r^{x}=\frac{d^{x}\left(z_{y}^{2}-z_{x}^{2}+1\right)-2 z_{x}\left(1+d^{y} z_{y}\right)}{\beta} \\
& r^{y}=\frac{d^{y}\left(z_{x}^{2}-z_{y}^{2}+1\right)-2 z_{y}\left(1+d^{x} z_{x}\right)}{\beta} \\
& r^{z}=\frac{z_{x}^{2}+z_{y}^{2}-1-2\left(d^{x} z_{x}+d^{y} z_{y}\right)}{\beta}
\end{aligned}
$$

Recall that each reflected ray originates from $\dot{O}[x, y, z(x, y)]$, we can compute the $[\sigma, \tau, u, v]$ ray coordinate of the ray by intersecting it with the $s t$ and $u v$ planes as:

$$
[\sigma, \tau, u, v]=\left[\frac{r^{x}}{r^{z}}, \frac{r^{y}}{r^{z}}, x-z \cdot \frac{r^{x}}{r^{z}}, y-z \cdot \frac{r^{y}}{r^{z}}\right]
$$

Substituting Equation (11) into Equation (12), we have

$$
\begin{aligned}
\sigma & =\frac{d^{x}\left(z_{y}^{2}-z_{x}^{2}+1\right)-2 z_{x}\left(1+d^{y} z_{y}\right)}{z_{x}^{2}+z_{y}^{2}-1-2\left(d^{x} z_{x}+d^{y} z_{y}\right)} \\
\tau & =\frac{d^{y}\left(z_{x}^{2}-z_{y}^{2}+1\right)-2 z_{y}\left(1+d^{x} z_{x}\right)}{z_{x}^{2}+z_{y}^{2}-1-2\left(d^{x} z_{x}+d^{y} z_{y}\right)} \\
u & =x-z \sigma, \quad v=y-z \tau
\end{aligned}
$$



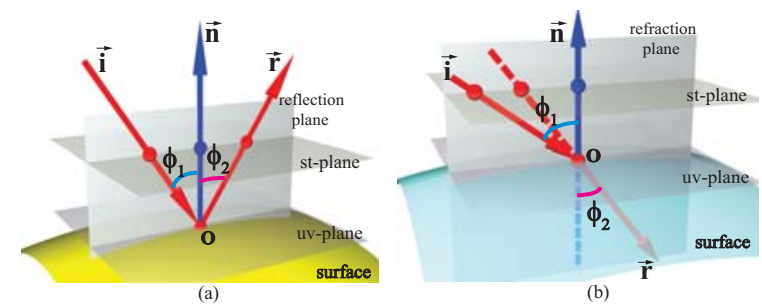

Figure 3. In reflection (a) and refraction (b), the incidence ray $\vec{i}$, the surface normal $\vec{n}$, and the exitance ray $\vec{r}$ are coplanar and satisfy Snell's Law. We choose the $u v$ parametrization plane to be the tangent plane at the point $\dot{O}$ we analyze. The st plane is a unit distance away from the $u v$ plane along $\vec{n}$.

This maps all reflected rays onto a ray manifold parameterized in $x$ and $y$. To analyze the local rays' behave, we can compute the local GLC characteristic equation (4). Since we are analyzing the ray behavior at $\dot{O}$, which is the origin of the coordinate system whose normal is $[0,0,1]$, we have

$$
z=z_{x}=z_{y}=0
$$

Substituting Equation (13) and (14) into the ray characteristic equation (4), we have:

$$
\begin{aligned}
A= & 4 \gamma\left(z_{x x} z_{y y}-z_{x y}^{2}\right) \\
B= & -2\left(z_{x x}+z_{y y}\right) \\
& -4 d^{x} d^{y} z_{x y}-2\left(\left(d^{x}\right)^{2} z_{x x}+\left(d^{y}\right)^{2} z_{y y}\right)
\end{aligned}
$$

where

$$
\gamma=\left(d^{x}\right)^{2}+\left(d^{y}\right)^{2}+1
$$

Recall that the Gaussian Curvature $K$ and the Mean Curvature $H$ of the Monge surface $z(x, y)$ can be computed as:

$$
\begin{aligned}
K & =\frac{z_{x x} z_{y y}-z_{x y}^{2}}{\left(z_{x}^{2}+z_{y}^{2}+1\right)^{2}} \\
H & =\frac{\left(1+z_{y}^{2}\right) z_{x x}-2 z_{x} z_{y} z_{x y}+\left(1+z_{x}^{2}\right) z_{y y}}{2\left(1+z_{x}^{2}+z_{y}^{2}\right)^{3 / 2}}
\end{aligned}
$$

At point $\dot{O}$, they can be simplified as:

$$
K=z_{x x} z_{y y}-z_{x y}^{2}, \quad H=\frac{1}{2}\left(z_{x x}+z_{y y}\right)
$$

Finally, if we assume the viewing direction is approximately the surface normal direction at $\dot{O}$, i.e., $d^{x} \approx d^{y} \approx 0$, we can further simplify the coefficients of the ray characteristic equation (15) as:

$$
\begin{aligned}
& A=4 z_{x x} z_{y y}-4 z_{x y}^{2}=4 K=4 \kappa_{1} \cdot \kappa_{2} \\
& B=-2\left(z_{x x}+z_{y y}\right)=-4 H=-2\left(\kappa_{1}+\kappa_{2}\right) \\
& C=1
\end{aligned}
$$

where $\kappa_{1}$ and $\kappa_{2}$ correspond to the min and max curvatures. Equation (19) indicates that we can directly compute the Gaussian and the Mean curvature using the ray characteristic equation of the local reflection GLC.
We can further substitute Equation (19) into the ray characteristic equation $A \lambda^{2}+B \lambda+C=0$ to solve for $\lambda$ as

$$
\lambda_{1}=\frac{1}{2 \kappa_{1}}, \quad \lambda_{2}=\frac{1}{2 \kappa_{2}}
$$

Yu and McMillan have recently shown that $\lambda_{1}$ and the $\lambda_{2}$ directly compute the caustic surface [17] of the reflected rays. Our analysis implies that these two caustic surfaces are closely related to the curvatures of the surfaces by a scale. This is mainly because under our approximation, the reflected direction is approximated $\left[2 z_{x}, 2 z_{y},-1\right]$, i.e., the $x$ and $y$ directional components double the normal $\left[z_{x}, z_{y},-1\right]$.

In addition, the coefficient $A$ in the characteristic equation reveals the directions of the all rays in the GLC [21]. When $A=0$, local rays will all be parallel to certain directions and they form a pushbroom camera [6]. Our analysis shows that $A=0$ indicates $K=0$. Therefore, since $K=0$ corresponds to the surfaces points are parabolic,local rays from an orthographic cameras will be reflected to form a pushbroom camera near the parabolic curves. This is consistent with Koenderink's observation [10] that specular reflections exhibit "duplications" at the parabolic points as "duplication" is a common image feature in pushbroom cameras.

\subsection{Refraction Ray Curvature}

Next, we apply a similar analysis to refractions. Assume the rays are refracted from the air into the surface, since the incident ray $\vec{i}$, the exit ray $\vec{r}$, and the normal $\vec{n}$ are coplanar, and the incident angle $\phi_{1}$ and exit angle $\phi_{2}$ satisfy Snell's Law (Figure 3(b)), we have:

$$
\sin \phi_{1}=m \sin \phi_{2}, \quad \vec{r} \cdot(\vec{i} \times \vec{n})=0
$$

where $m$ is the refractive index of the refraction surface.

Furthermore, we have

$$
\cos \phi_{1}=\frac{\vec{i} \cdot \vec{n}}{|\vec{i}| \cdot|\vec{n}|}, \quad \cos \phi_{2}=\frac{\vec{r} \cdot \vec{n}}{|\vec{r}| \cdot|\vec{n}|}
$$

For near-flat surfaces with an orthogonal viewing direction, the incidence angle $\phi_{1}$ is relatively small. Thus, we can approximate the refracted direction as:

$$
\vec{r} \approx \frac{1}{m} \cdot \hat{i}+\frac{m-1}{m} \cdot \hat{n}
$$

where $\hat{i}$ and $\hat{n}$ are normalized vectors of $\vec{i}$ and $\vec{n}$.

Substituting Equation (7) and (8) into Equation (23), we obtain the refracted direction $\vec{r}=\left[r^{x}, r^{y},-1\right]$ as:

$$
\begin{aligned}
r^{x} & =\frac{z_{x}(m-1) \sqrt{\gamma}+d^{x} \sqrt{\beta}}{(m-1) \sqrt{\gamma}+\sqrt{\beta}} \\
r^{y} & =\frac{z_{y}(m-1) \sqrt{\gamma}+d^{y} \sqrt{\beta}}{(m-1) \sqrt{\gamma}+\sqrt{\beta}}
\end{aligned}
$$


We then compute the $u v$ coordinates of the ray by intersecting it with the $u v$ plane similar to Equation (12), and we have the $[\sigma, \tau, u, v]$ coordinate of the refracted ray as:

$$
\begin{aligned}
\sigma & =-r^{x}, & \tau & =-r^{y} \\
u & =x+z r^{x}, & v & =y+z r^{y}
\end{aligned}
$$

Finally, we can compute the ray characteristic equation of the refraction GLC using Equation (4) by taking partial derivatives of $\sigma, \tau, u$, and $v$ with respect to $x$ and $y$ in Equation (25). Since we choose the surface tangent plane as the parametrization plane, we can further simplify the $A, B$, and $C$ coefficients using Equation (14) as:

$$
\begin{aligned}
A & =\frac{\gamma(m-1)^{2}\left(z_{x x} z_{y y}-z_{x y}^{2}\right)}{\left(1+(m-1) \sqrt{\gamma}^{2}\right.} \\
B & =\frac{\sqrt{\gamma}(m-1)\left(z_{x x}+z_{y y}\right)}{1+(m-1) \sqrt{\gamma}}
\end{aligned}
$$

By further assuming the viewing direction is orthogonal to the surface, i.e., $d^{x}=d^{y}=0$, we have:

$$
\begin{aligned}
& A=\left(\frac{m-1}{m}\right)^{2}\left(z_{x x} z_{y y}-z_{x y}^{2}\right)=\left(\frac{m-1}{m}\right)^{2} K \\
& B=\frac{(m-1)}{m}\left(z_{x x}+z_{y y}\right)=\frac{2(m-1)}{m} H \\
& C=1
\end{aligned}
$$

Similar to the reflection curvature analysis, Equation (27) implies that we can compute the Gaussian and the Mean Curvature directly from the $A, B$, and $C$ coefficients of the refraction GLC. Furthermore, we can solve the ray characteristic equation for $\lambda$ as:

$$
\lambda_{1}=\frac{m}{(m-1) \kappa_{1}}, \quad \lambda_{2}=\frac{m}{(m-1) \kappa_{2}}
$$

This indicates that the refraction caustic surfaces are related to the curvature of the near-flat mirror surfaces by a scale determined by the refractive index.

\section{Estimating the Local GLCs}

In this section, we show how to estimate the local GLCs of specular surfaces from their images.

\subsection{Pixel-Pattern Correspondences}

In order to estimate the local GLC, we first compute the rays exiting from the surface. To do so, we place a known pattern (e.g., a checkerboard) in front of the reflective surface or beneath the refractive surface and capture an image of the surface. We then find the pixel-pattern correspondences from the image.

For reflective surfaces such as mirrors, we use a colorcoded checkerboard pattern to reduce the ambiguities between neighboring corners. Since the distortion patterns on the reflective surface may dramatically change, we use
Scale Invariant Feature Transform (SIFT) to detect the feature points. We then perform global matching using SSD on all the three color channels to find the potential matching pairs. We also assume the topology of the grid does not change to remove the outliers.

For refractive surfaces such as water surfaces that evolve over time, we implement an iterative method similar to the one used in [12] to update the pixel-to-pattern correspondences over the frames. Specifically, we assume that the water surface is undisturbed at time $\mathrm{t}=0$ and we use Harris corner detector to detect corners for establishing the initial pixel-to-pattern correspondences. Then we propagate them through time using optical flow estimation.

\subsection{Ray-Pixel Correspondence}

Once we compute the pixel-to-pattern correspondence, we set out to compute the reflection or the refraction rays at the corresponding pixels. Since our focus is to model near-flat surfaces, we find, from our experiments, it is usually sufficient to simply approximate the surface as a plane. Therefore, for reflection surfaces, we simply calibrate its attached background surface to determine this plane. For refractions, we capture a pair of images of the pattern, one without the fluid surface and one with the fluid surface with no disturbances. Since the pattern will drift due to refractions, we then measure the height of the fluid surface in terms of the amount of drifting.

\subsection{Estimating the Local GLC}

Notice that since we only estimate the corresponding rays at the corners, we only obtain a sparse set of rays. To further improve the quality of the estimation, we first run Delaunay triangulations on the detected corners. We warp the corner rays into the ray space and linearly interpolate the $[\sigma, \tau, u, v]$ coordinates for each pixel by rasterizing the triangles. This provides us with densely sampled rays. To estimate the GLC model at each pixel, we pick the four neighboring rays and find the optimal GLC that best fits these rays.

Assume the rays are represented as: $\vec{r}_{k}=$ $\left[\sigma_{k}, \tau_{k}, u_{k}, v_{k}\right], k=1 \ldots 5$, we use the canonical form [21] to specify the target GLC. The canonical GLC uses the three generator rays that have form: $\left[\tilde{\sigma_{1}}, \tilde{\tau_{1}}, 0,0\right]$, $\left[\tilde{\sigma_{2}}, \tilde{\tau_{2}}, 1,0\right]$, and $\left[\tilde{\sigma_{2}}, \tilde{\tau_{2}}, 0,1\right]$.

For every ray $\overrightarrow{r_{k}}$ in a GLC, it can be written as some affine combination of the three generator rays as:

$$
\begin{array}{r}
\vec{r}_{k}\left[\sigma_{k}, \tau_{k}, u_{k}, v_{k}\right]=\left(1-\alpha_{k}-\beta_{k}\right) \cdot\left[\tilde{\sigma_{1}}, \tilde{\tau_{1}}, 0,0\right] \\
\alpha_{k} \cdot\left[\tilde{\sigma_{2}}, \tilde{\tau_{2}}, 1,0\right]+\beta_{k} \cdot\left[\tilde{\sigma_{3}}, \tilde{\tau_{3}}, 0,1\right]
\end{array}
$$

It is easy to see that the affine coefficients can be computed as $\alpha_{k}=u_{k}$ and $\beta_{k}=v_{k}$, and we can rewrite Equation (29) as: 


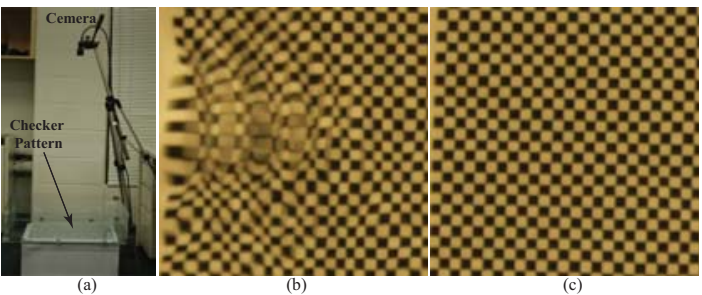

Figure 4. Capturing Near-flat Refractive Surfaces. (a) show the setup of our system. (b) shows the distorted checkerboard pattern. (c) captures the initial fluid surface without disturbance.

$$
\begin{aligned}
\sigma_{k} & =\left(1-u_{k}-v_{k}\right) \tilde{\sigma_{1}}+u_{k} \tilde{\sigma_{2}}+v_{k} \tilde{\sigma_{3}} \\
\tau_{k} & =\left(1-u_{k}-v_{k}\right) \tilde{\tau_{1}}+u_{k} \tilde{\tau_{2}}+v_{k} \tilde{\tau_{3}}
\end{aligned}
$$

Therefore each ray maps to two linear constraints. Recall that the canonical GLC is specified by six variables. Since we picked five rays, we form an over-determined system as:

$$
\left(\begin{array}{cc}
S & T
\end{array}\right)=M \cdot\left(\begin{array}{cc}
\tilde{\sigma_{1}} & \tilde{\tau_{1}} \\
\tilde{\sigma_{2}} & \tilde{\tau_{2}} \\
\tilde{\sigma_{3}} & \tilde{\tau_{3}}
\end{array}\right)
$$

where

$$
\begin{aligned}
S= & \left(\begin{array}{l}
\sigma_{1} \\
\vdots \\
\sigma_{5}
\end{array}\right), \quad T=\left(\begin{array}{l}
\tau_{1} \\
\vdots \\
\tau_{5}
\end{array}\right) \\
M= & \left(\begin{array}{lll}
1-u_{1}-v_{1} & u_{1} & v_{1} \\
\vdots & \vdots & \vdots \\
1-u_{5}-v_{5} & u_{5} & v_{5}
\end{array}\right)
\end{aligned}
$$

To find the optical GLC, we simply use the Singular Value Decomposition (SVD) to solve for Equation (31).

\section{Experiments and Results}

For synthetic surfaces, we use the PovRay Ray Tracer to render the image of the surface viewed from an orthographic camera [14]. For real surfaces, we capture a distorted image on the surface from a camera positioned relatively faraway form the surface to simulate the orthographic viewing direction.

\subsection{Synthetic Surfaces}

We first conduct experiments using our method on known parametric surfaces. In Figure 6, we show two nearflat parametric surfaces: the top row is a combination of Gaussians and the bottom row is a scaled dimple surface. We show the color-coded height fields, the Gaussian curvature fields, and the mean curvature fields. In column 2, we show the ray-traced images of the surfaces viewed from an orthographic camera.

We then use the algorithm described in Section 4 to estimate the Gaussian and the Mean Curvature. In column 4 and 6, we show the estimated Gaussian and Mean curvature. Our ray-curvature method accurately recovers the curvatures. In Figure 7, we apply the same method on two refractive surfaces. We assume the surface has a refractive index of 1.33 and has a uniform height of 1.5. Despite the approximation in Equation (23), our method still faithfully capture the curvature characteristics on these refractive surfaces.

\subsection{Real Surfaces}

Using our method, we have also experimented with capturing high-order differential geometry attributes on real reflection and refraction surfaces.

Our first experiment is to recover the geometry of a transparent paper, as shown in Figure 5. We stick the paper on a black board and the paper becomes highly reflective. We then place a colored checkerboard in front of the paper. The checkerboard is positioned approximately parallel to the white board to minimize distortions and the camera is placed relative far away from the slip. We use a second camera to calibrate the position of the checkerboard and apply the method described in Section 4 to compute the pixelto-pattern correspondences. We assume the paper lies on a plane and we use the known checkerboard position and the correspondences to compute the reflected ray at each corner.

In Figure 5, we show the recovered curvatures of the transparent paper. Notice that the recovered curvature fields are closely related to the degree of distortions on the image. Larger Gaussian curvature correspond to stronger distortions: the aspect ratio of the pattern is severely distorted at high Gaussian curvature regions. These characteristics are difficult to model using traditional methods that only recover the vertices and normals.

To capture the curvature on the water's surfaces, we set up a single-camera system as shown in Figure 4. We place a Dragonfly Flea camera high above the water surface to synthesize an orthographic viewing camera. We first calibrate the camera and the checker pattern without water. We then take an image of the undisturbed water surface and recalibrate the pattern. The translation of the pattern provides us the height of the surface. We then estimate the pixel-to-ray correspondence at each frame as described in 4, find the optimal GLC, and compute the Gaussian and Mean curvature. In Figure 8, we show the recovered Gaussian and mean curvature fields of the water surface captured at four different time instance. Notice that the curvature fields identify important shape characteristics related to the distortions.

In our experiment, we find that it is often difficult to establish one-to-one correspondences from the checkerboard corners to the observed pattern, especially for fluid surfaces, mainly due to the motion blur, image distortions, and illumination inconsistencies. As a result, our method produces a sparse set of correspondences and we rely on the Delaunay Triangulation to interpolate the ray coordinates for each pixel. 


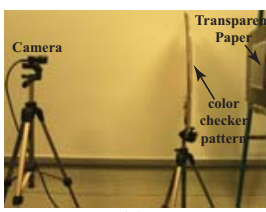

(a)

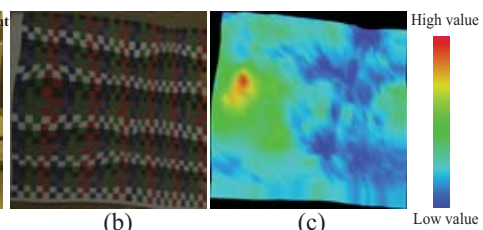

(b)
Figure 5. Capturing Near-flat Reflection Surfaces. (a) shows our
system setup. We mount a transparent paper on the blackboard and place the colored checkerboard parallel to the blackboard. (b) shows the captured distortion image. (c) shows the estimated Gaussian curvature using our method.

\section{Conclusions}

We have presented a new framework for modeling and capturing shape characteristics on near-flat specular (refractive and reflective) surfaces. Unlike traditional methods that focus on reconstructing the surface positions and normals, our framework directly recovers the higher-order geometry attributes such as the curvature on specular surfaces. To do so, we model the local reflections and refractions in terms of general linear cameras (GLCs). We have derived a new theory that correlates the higher-order differential geometry attributes with the local GLC intrinsics. Specifically, we have shown that the Gaussian and Mean Curvature can be directly computed in terms of the local reflection or refraction GLC's characteristic equation.

We have validated our ray-curvature theory on both synthetic and real-world specular surfaces. Our method places a known pattern in front of the reflective surface or beneath the refractive surface and captures a distorted image on the surface. We then compute the optimal GLC using a sparse set of correspondences and estimate the curvatures from the GLC. Experiments have demonstrated that our methods are robust and highly accurate.

In the future, we plan to explore how to extend our raycurvature theory to model a broader class of surfaces. The key difficulty is establishing pixel-ray correspondences. We resolve this correspondence problem by assuming that the surface is near-flat and can be approximated by a plane. We would like to investigate how to directly use the GLC distortions to model the ray structures without requiring the pixel-to-ray correspondences.

\section{Acknowledgement}

This work has been supported by the National Science Foundation under grant NSF-MSPA-MCS-0625931.

\section{References}

[1] A. Blake. Specular stereo. In Proc. of the Ninth International Joint Conference on Artificial Intelligence, pages 973-976, 1985. 2

[2] T. Bonfort and P. Sturm. Voxel carving for specular surfaces. In ICCV '03, page 591. 2
[3] T. Bonfort, P. Sturm, and P. Gargallo. General specular surface triangulation. In $A C C V$, volume II, pages $872-881$, jan 2006. 2

[4] D. Enright, S. Marschner, and R. Fedkiw. Animation and rendering of complex water surfaces. In SIGGRAPH '02, pages 736-744, 2002. 1

[5] W. C. K. B. L. Gotwols. Two-dimensional optical measurement of wave slope. In Applied Optics, pages 3476-3478, Nov 1983. 1

[6] R. Gupta and R. I. Hartley. Linear pushbroom cameras. IEEE Trans. Pattern Anal. Mach. Intell., 19(9):963-975, 1997. 4

[7] K. Ikeuchi. Determining surface orientations of specular surfaces by using the photometric stereo method. pages 268 276, 1992. 2

[8] M. M. W. H. Ivo Ihrke, Kyros Kutulakos and H. Lensch. Transparent and reflective scene reconstruction. EUROGRAPHICS STAR - State of The Art Report, May 2008. 2

[9] H. Z. J. Dai, J. Kim and X. Gu. Visualizing the evolutions of silhouettes. In Computer Graphics International '07. 2

[10] J. J. Koenderink. Solid shape. MIT Press, 1990. 1, 2, 4

[11] K. N. Kutulakos and E. Steger. A theory of refractive and specular $3 \mathrm{~d}$ shape by light-path triangulation. In ICCV '05, pages $1448-1455,2005.2$

[12] N. J. W. Morris and K. N. Kutulakos. Dynamic refraction stereo. In ICCV '05, pages 1573-1580. 2, 5

[13] H. Murase. Surface shape reconstruction of an undulating transparent object. In Radiometry, pages 213-217, 1992. 2

[14] Pov-Ray. http://www.povray.org/. 6

[15] A. C. Sanderson, L. E. Weiss, and S. K. Nayar. Structured highlight inspection of specular surfaces. 10(1):44-55, 1988. 2

[16] S. Savarese and P. Perona. Local analysis for 3d reconstruction of specular surfaces - part ii. In $E C C V^{\prime} 02$, pages 759 774. 2

[17] R. Swaminathan, M. Grossberg, and S. Nayar. Caustics of catadioptric cameras. In $I C C V$, volume 2 , pages $2-9$, Jul 2001. 2, 4

[18] R. Swaminathan, M. D. Grossberg, and S. K. Nayar. Nonsingle viewpoint catadioptric cameras: Geometry and analysis. IJCV, 66(3):211-229, 2006. 2

[19] M. Tarini, H. P. A. Lensch, M. Goesele, and H.-P. Seidel. $3 \mathrm{~d}$ acquisition of mirroring objects using striped patterns. Graphical Models, 67(4):233-259, 2005. 2

[20] J. Wang and K. J. Dana. A novel approach for texture shape recovery. In ICCV '03, pages 1374-1380. 2

[21] J. Yu and L. McMillan. General linear cameras. In ECCV, pages 14-27, 2004. 2, 3, 4, 5

[22] J. Yu and L. McMillan. Modelling reflections via multiperspective imaging. In Proc. IEEE CVPR, June 2005. 2

[23] X. Zhang and C.S.Cox. Measuring the two-dimensional structure of a wavy water surface optically: a surface gradient detector. In Experiments in Fluids, pages 225-237, 1994. 2

[24] L. Zhou, C. Kambhamettu, and D. Goldgof. Fluid structure and motion analysis from multi-spectrum $2 \mathrm{~d}$ cloud image sequences. In Proc. IEEE CVPR, pages 744-751, 2000. 1 


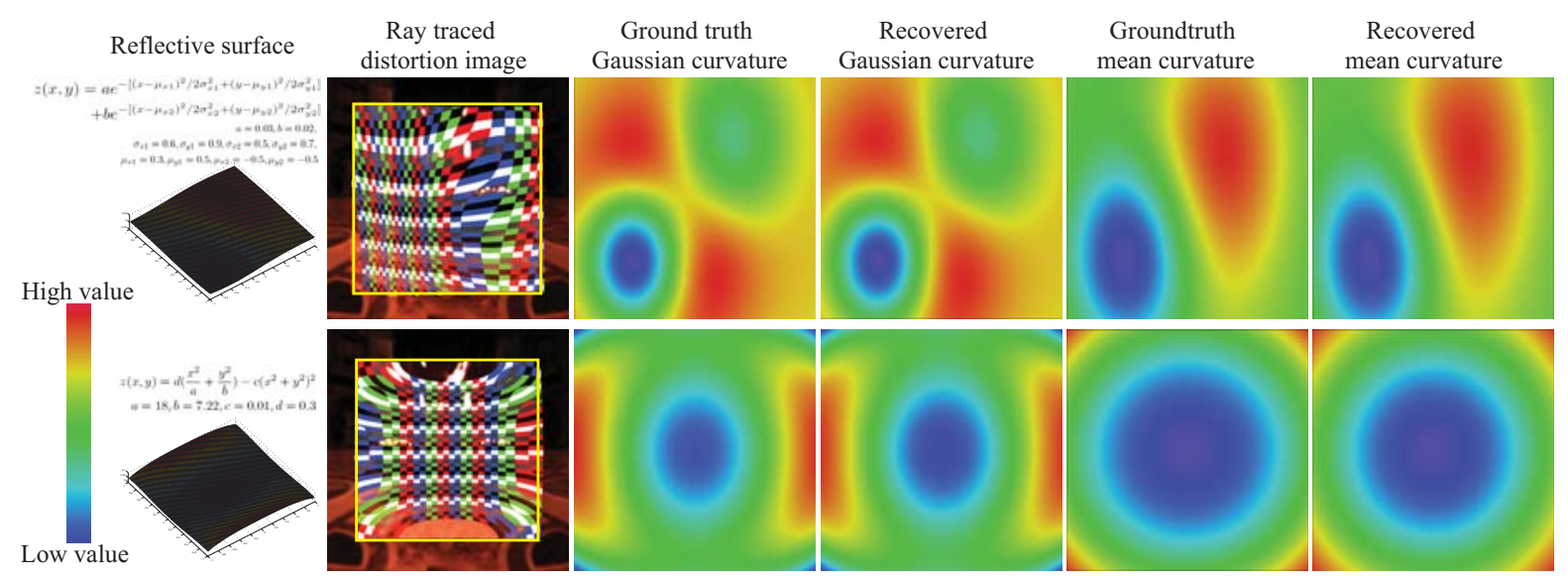

Figure 6. The recovered Gaussian and mean curvature using ray-traced reflection images.

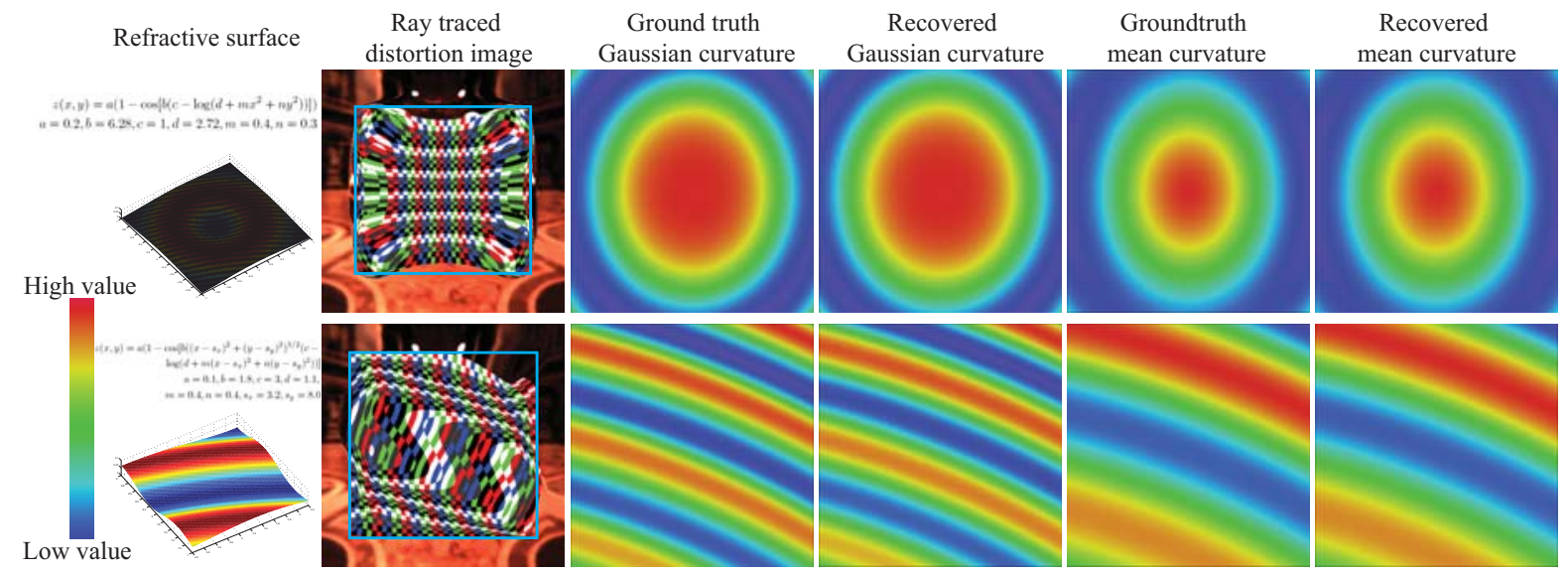

Figure 7. The recovered Gaussian and mean curvature using ray-traced refraction images.

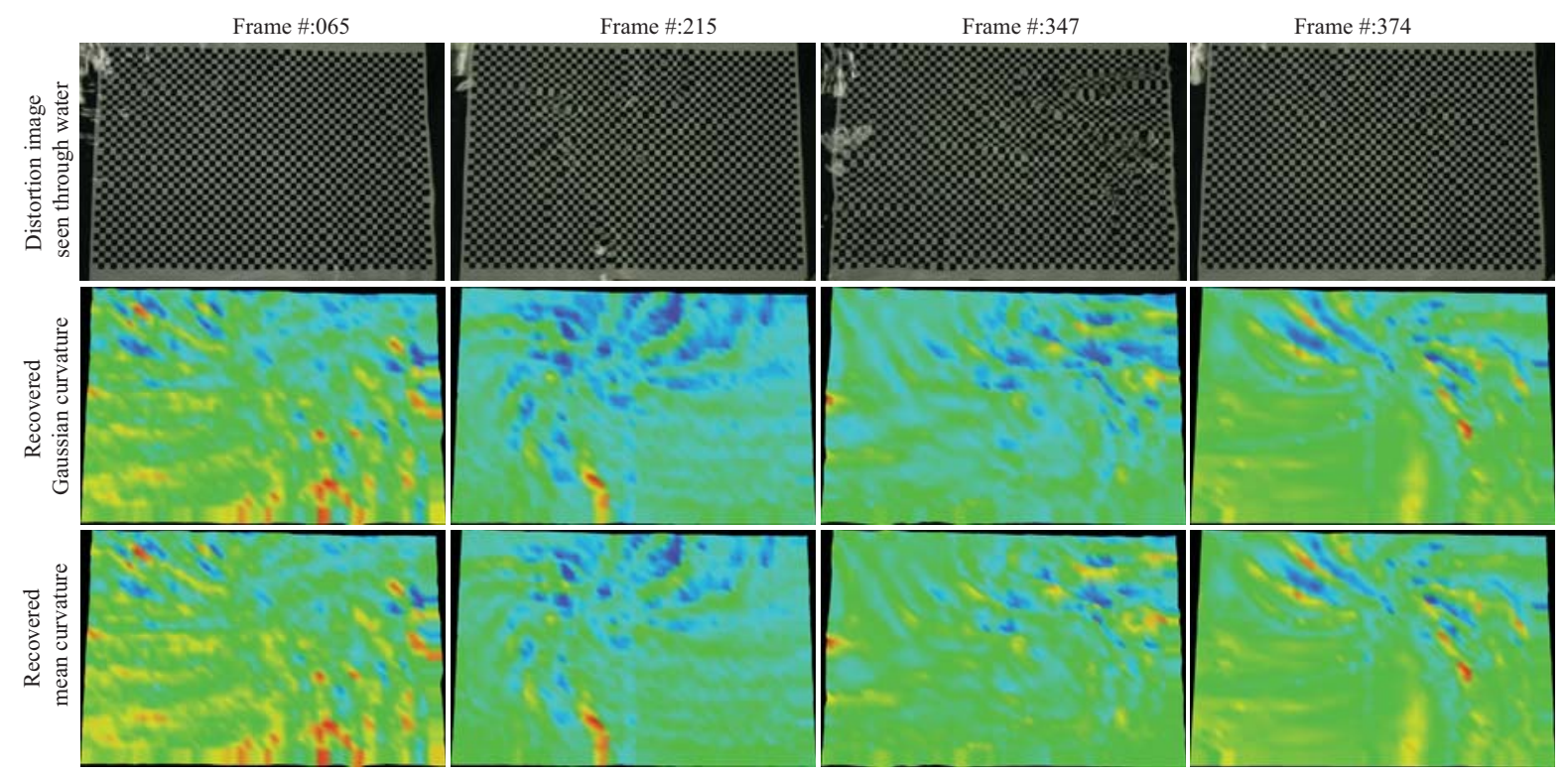

Figure 8. The recovered Gaussian and mean curvature of the dynamic fluid surface at different time instances. 